1. Autonomie und Verantwortbarkeit in digitalen Machtund Herrschaftsmechanismen 


\section{Digitale Subjektivierungsmacht als Technologie des Selbst und Digitales Selbst}

Eine zweifache machttheoretische Annährung an das Subjekt der Privatheit

Julia Valeska Schröder

\section{Einleitung}

Die im Web 2.0 aufgeworfene Privatheitsproblematik und der für sie konstitutive Dualismus von Öffentlichkeit/Privatheit hängt untrennbar mit der im Subjekt selbst eingeschriebenen Spannung zwischen Individuum und Gesellschaft zusammen. Bei beiden Dualismen handelt es sich um Gegenüberstellungen, die in ihrer Schematik - von kritischen und vor allem feministischen Theorien - bereits in den 1980er Jahren grundlegend hinterfragt wurden. Denn die Idee des liberalen Subjekts beruht auf der Annahme einer starken Dichotomie von Individuum und Gesellschaft, von Öffentlichkeit und Privatheit und zeigt sich oft in subjekttheoretischen Betrachtungen des sonst sehr heterogenen Privatheitsdiskurses. Mit dem gängigen Verständnis von Privatheit als selektiver Kontrolle des Zugangs zum Selbst wird dezidiert und implizit die Annahme eines unabhängigen, handlungsmächtigen, seinen sozialen Praktiken vorhergehenden Subjekts reproduziert. Der normative Referenzrahmen von Selbstbestimmtheit, Verantwortung, individueller Handlungsfähigkeit, Entscheidungsmacht, Autonomie und Souveränität als Aspekte des Privaten ist hierbei weit verbreitet. Es handelt sich dabei oft um die Vorstellung eines Subjekts, dem diese Eigenschaften essentialistisch zugeschrieben werden, das sich preisgeben und sein Inneres offenlegen oder dazu genötigt werden kann - die unfixierbare Offenheit seines Wesens, seine gesellschaftliche Verwobenheit und existentielle soziale Abhängigkeit rückt dabei aus dem Fokus der Betrachtungen. Julie Cohen diagnostiziert dem Privatheitsdiskurs deshalb eine Distanzierung gegenüber dem grundlegend gesellschaftlichen, sozial konstruierten Subjekt und kritisiert, dass die Analysen des Subjektivierungsprozesses und der Bedingungen der Subjektkonstitution vernachlässigt 
werden. ${ }^{1}$ Eine privatheitstheoretische Vernachlässigung von kritischen Theorien der sozialen, positiven Freiheit (im Gegensatz zum liberalen Konzept der individuellen, negativen Freiheit) würde eine „deep conceptual poverty about what selves are made of " 2 bedeuten.

Allerdings zeigt nicht zuletzt Cohens Kritik, dass das liberale, autonome Subjekt im Rahmen der Privatheitsforschung auch zunehmend zugunsten eines sozial eingebetteten Subjekts angefochten wird. ${ }^{3}$ Theoretikerinnen der intersubjektiven Privatheitstheorie, beispielsweise Helen Nissenbaum ${ }^{4}$ sowie Leysia Palen und Paul Dourish ${ }^{5}$ zeigen auf, wie soziale Beziehungen, gesellschaftliche Kontexte, Medienpraktiken und kulturelle Praktiken aufs Engste mit dem Privatheitssubjekt verwoben sind. Aber auch mit der Annahme eines in kontextualisierten Netzwerken verwickelten, sozialen Subjekts, das als individuelle Einheit seine Privatheit reguliert, ${ }^{6}$ stehen weiterhin nicht die für die Subjektivierung konstitutiven Machtdynamiken und die gesellschaftstheoretische Perspektive im Vordergrund, die durch kritische Subjektivierungstheorien hervorgehoben werden sollen.

Die wichtige Kritik an der Dominanz liberaler Subjekttheorie im Privatheitsdiskurs will ich deswegen mit einer dezidiert machttheoretischen Pointe hervorheben. Die Betrachtung von Subjektivierungsprozessen ist für den Privatheitskontext aus zwei Gründen relevant: Sie wirft erstens Licht auf die Problematik einer in liberaler, politischer Theorie verhafteten Privatheitstheorie und stellt zweitens die gesellschaftspolitische Frage nach den Entstehungsbedingungen des Subjekts, seinen Konstitutionsprozessen und prägenden Machtmechanismen sowie mit ihm verwickelten Herrschaftsverhältnissen. Den kritischen subjekttheoretischen Blick will ich im Folgenden auf die gesellschaftlichen Strukturveränderungen durch das Web 2.0 werfen. Denn diese führen nicht nur zu einer zunehmenden Veränderung und Einschränkung der Privatheit, sondern bringen zugleich eine Veränderung der Bedingungen von Subjektivierungsprozessen mit sich und damit neue Subjektformen hervor. Ein Phänomen, das ich als digitale Subjektivierung bezeichnen werde, kommt durch die „operating conditions of digital-infrastructures ${ }^{\text {"7 }}$ zustande, die Teil der "functioning con-

1 Vgl. Cohen (2012).

2 Ebd. (S. 6).

3 Vgl. exemplarisch Decew (2015); Bennett (2011); Cohen (2012).

4 Vgl. Nissenbaum (2010).

5 Vgl. Palen/Dourish (2003).

6 Vgl. ebd.

7 Couldry/Hepp (2017: S. 161). 
ditions of the self" ${ }^{* 8}$ sind. Die veränderten Kommunikationsmedien und medialen Aufforderungen zur Selbstdarstellung9 ${ }^{9}$ und digitaler Sozialisation $^{10}$, wie sie bspw. in sozialen Netzwerken praktiziert werden, sind für eine Betrachtung eines Subjekts der Privatheit, das aus kommunikativen Praktiken überhaupt erst hervorgeht, von größter Bedeutung. ${ }^{11}$ Ich stelle deswegen die Frage, wie digitale Subjektivierungsmacht theoretisch zu greifen ist und wie sich der Privatheitsproblematik gesellschafts- und machttheoretisch über Subjektivierungstheorien genähert werden kann.

Um das Phänomen digitaler Subjektivierungsmacht kritisch zu beleuchten, werde ich zwei Ansätze zu digitalen Subjektivierungsprozessen anhand der Konzepte Technologie des Selbst ${ }^{12}$ und Digitales Selbst ${ }^{13}$ herausarbeiten, um diese miteinander und gegeneinander zu lesen. Dabei will ich zeigen, dass das Zusammendenken der mit ihnen aufgedeckten Machtbeziehungen und Herrschaftsdynamiken wichtig für eine kritische Analyse von digitalem Privatheitsmanagement sowie für die Ausrichtung der Machttheorien an aktuellen Problematiken der zunehmend digitalisierten Gesellschaft ist. Zuerst werde ich dafür post-strukturalistische Ansätze der Subjektivierungsmacht von Louis Althusser ${ }^{14}$, Michel Foucault ${ }^{15}$ und Judith Butler ${ }^{16}$ mobilisieren, um die liberale Subjektillusion und die zum Teil wenig vielschichtige Machtanalyse des Privatheitsdiskurses mit einer gesellschaftstheoretischen Lesart des Subjekts und der post-strukturalistischen Einsicht des zentralen Funktionsmechanismus von Macht als Produktion von Subjektivität zu konfrontieren. Auf dieser Grundlage werde ich eine post-strukturalistisch geprägte Perspektive auf digitale Subjektivierungsmacht als Technologie des Selbst herausarbeiten und damit die machttheoretische Ambivalenz des Subjekts hervorheben. Anschließend greife ich die dabei entstehende Problematik der Überbetonung des Diskursiven auf und nähere mich dem Phänomen digitaler Subjektivierung mit dem in der Privatheitstheorie etablierten Konzept des Digitalen Selbst. Somit ergänze ich dieses um eine herrschaftskritische, mediensensible Problematisierung digitaler Subjektivierungsmacht. Diese setzt der post-strukturalisti-

\section{Ebd.}

9 Vgl. Bucher (2012a); Sauter (2014).

10 Vgl. Bucher (2012b).

11 Vgl. Gentzel et al. (2019).

12 Vgl. Paulitz (2014); Bublitz (2014); Sauter (2014).

13 Vgl. u. a. Graham/Wood (2003); Cheney-Lippold (2011).

14 Vgl. Althusser (2010).

15 Vgl. Foucault (1989); Foucault (2005).

16 Vgl. Butler (2001). 
schen Betonung der produktiven Macht digitaler Subjektivierung ein Gegengewicht, indem sie digitale Objektivierungsprozesse von analogen differenziert und eine souveräne, repressive Machtform sichtbar werden lässt. Abschließend werde ich versuchen, diese konträren Perspektiven konstruktiv zu verbinden, d. h. die Zusammenhänge von Herrschaftstechnologie und Selbsttechnologie nachzuzeichnen und die Relevanz einer verdoppelten Subjektivierungsmacht im Kontext digitaler Medien und der Privatheitsproblematik aufzuzeigen.

\section{Subjektivierungsmacht}

Den Prozess der Subjektivierung post-strukturalistisch als Phänomen der Macht zu begreifen, wird auf theoretischer Ebene mit Althussers Konzeption der Anrufung ${ }^{17}$, Foucaults Analytik der Macht ${ }^{18}$ und schließlich durch Butlers Psyche der Macht ${ }^{19}$ möglich. Sie alle richten ihre Aufmerksamkeit auf den politisch bedeutsamen Status des Subjekts, der „die sprachliche Gelegenheit des Individuums, Verständlichkeit zu gewinnen und zu reproduzieren, also die sprachliche Bedingung seiner Existenz und Handlungsfähigkeit“ ${ }^{20}$ beschreibt. Post-strukturalistische Subjektivierungstheorien verweigern sich derjenigen humanistisch-liberalen Tradition, die den bereits subjektivierten Menschen als unhinterfragten Ausgangspunkt subjekttheoretischer Überlegungen nimmt. Sie kritisieren die Idee des autonomen Subjekts als „eine irreduzible Instanz der Reflexion, des Handelns und des Ausdrucks, welche ihre Grundlage nicht in den kontingenten äußeren Be-

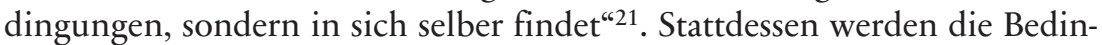
gungen untersucht, die Individuen gesellschaftlich handlungsfähig machen und zu anerkannten Subjekten werden lassen. Die Grundannahme hierbei ist, dass der Mensch „ein Produkt fortdauernder anthropologischer Projektionen, Programmatiken und Selbstformungen “22 ist und, dass Subjekte somit historisch kontingent und nur in Abhängigkeit von gesellschaftlichen, institutionellen Machtstrukturen zu erfassen sind. Insofern „beschreibt der Begriff des Selbst nicht eine stabile Existenzweise oder eine Identität, die über einen Ursprung verfügte oder der eine Teleologie zukä-

17 Vgl. Althusser (2010).

18 Vgl. Foucault (2005).

19 Vgl. Butler (2001).

20 Ebd. (S. 15).

21 Reckwitz (2008: S. 12).

22 Wiede (2014). 
me, sondern ein relationales Feld, das seine eigenen Transformationen her-

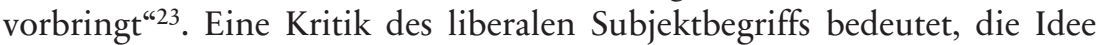
der Individualität des Subjekts zu brechen: Das Individuum hat keine gesellschaftliche Existenz; zugleich kann das Subjekt nicht im engeren Sinne als individuell verstanden werden. Das Subjekt ist weder feststehendes Wesen noch innere Substanz, sondern ein Prozess, in dem der ,äußerliche Kontext internalisiert und inkorporiert wird: Gesellschaftliche Strukturen schreiben sich über diskursive Prozesse in die Individuen ein, welche diese Strukturen reproduzieren und auch umdeuten können. Somit ist die Beziehung zum Selbst für das Subjekt stets eine Beziehung zu Anderen, wobei das Subjekt zum Objekt eines Gegenübers werden kann (und umgekehrt). ${ }^{24}$

Der Begriff ,Subjekt' hat in diesem Kontext zwei Bedeutungen: einerseits das Subjekt, das der Herrschaft einer anderen unterworfen ist und in ihrer Abhängigkeit steht. Andererseits dasjenige, das durch Konstruktionen durch sich selbst und von außen mit einer eigenen Identität versehen ist und mit ihr verhaftet bleibt. ${ }^{25}$ Beide Varianten beziehen sich nach Foucault auf eine Form von Macht, die unterjocht und unterwirft (auf Englisch wegen des gemeinsamen Wortstamms besonders deutlich: „a form of power, which subjugates and makes subject to"26). Subjekte sind in dieser Lesart Effekte von Machtverhältnissen und stellen wiederum selbst Machtsubjekte dar, von denen eine Wirkungsmacht und Handlungsfähigkeit ausgeht. Das Individuum, das zur Subjektwerdung strebt, wird in diesem Entstehungsprozess notwendigerweise denjenigen Machtbeziehungen unterworfen, die strukturell die gesellschaftliche Organisation prägen. Subjektivierungsmacht ist nach Foucault eine Machtform, die Menschen in Subjekte verwandelt; sie wirkt auf und durch das Individuum. ${ }^{27}$

Man darf das Individuum, denke ich, also nicht als eine Art elementaren Kern, primitives Atom, vielfältige und träge Materie begreifen, auf die die Macht angewendet wird, gegen welche sie sich richtet und die die Individuen unterwerfen und brechen würde.

23 Reigeluth (2015: S. 26).

24 Vgl. Butler (2001).

25 Vgl. Foucault (2005: S. 245).

26 Foucault (1982: S. 781). Um - wie im Englischen - den Unterwerfungsprozess der Subjektivierung hervorzuheben, wird dieser als Subjektivation ins Deutsche übersetzt.

27 Vgl. ebd. (S. 245). 
In Wirklichkeit ist das, was bewirkt, dass Körper, Gesten, Diskurse, Wünsche als Individuen [lies im Folgenden: Subjekte28; Anm. J.V.S.] identifiziert und konstituiert werden, eine der ersten Wirkungen der Macht. Das Individuum ist also nicht das Gegenüber der Macht; es ist eine ihrer ersten Wirkungen. Das Individuum ist ein Machteffekt und gleichzeitig, in genau dem Maße, wie es eine ihrer Wirkungen ist, verbindendes Element. ${ }^{29}$

Diese Machtform ist aus post-strukturalistischer Perspektive eine existenzielle soziale Dynamik, die Subjekte mittels diskursiver Produktivität bildet und formt. ${ }^{30}$ Sie ,gilt dem unmittelbaren Alltagsleben, das die Individuen in Kategorien einteilt, ihnen ihre Individualität zuweist, sie an ihre Identität bindet und ihnen das Gesetz einer Wahrheit auferlegt, die sie in sich selbst und die anderen in ihnen zu erkennen haben". ${ }^{31}$ Es vollzieht sich eine „Objektivierung“ ${ }^{\prime 32}$ von Individuen, indem das Subjekt „in sich selbst geteilt oder von den anderen unterschieden und getrennt ${ }^{\text {"33 }}$ wird. Dies sind die notwendigen Unterwerfungen zur Erlangung von Subjektivität verstanden als ,auf Unterscheidung und auf Unterteilung angelegte Praktiken“34. Die Unterwerfung vollzieht sich nach Foucault durch kategoriale Identitätseinordnung; der normalisierende Zugehörigkeitszwang zu einer sozialen Gruppe sichert das soziale Leben, stellt aber gleichzeitig eine machtvolle Klassifizierung und Hierarchisierung dar. Individuen sind in ihrem Streben nach einer gesellschaftlichen Existenz der unterwerfenden Subjektivierungsmacht als Existenzbedingung und als Bedingung ihres Fortbestandes ständig ausgesetzt. Subjektivierung im Kern als Unterwerfung oder als Dezentrierung anzusehen, kann durch Althussers Konzept der Anrufung vermittelt werden, insofern die ,gesellschaftliche Konfiguration von Subjekten im Zusammenwirken von Fremd- und Selbstformierung denkbar“ 35 wird. In der Anrufung (Interpellation) formiert sich das

28 Foucaults Begriffsverwendung ist teilweise inkonsequent. Um Unklarheit entgegenzuwirken, orientiert sich dieser Text an Butlers Begriffsdifferenzierung zwischen Subjekt und Individuum, nach der „Individuen den Ort des Subjektes [besetzen; Anm. J.V.S.], ihre Verständlichkeit erst durch ihre Subjektwerdung [erlangen; Anm. J.V.S.]“. Butler (2001: S. 16).

29 Foucault (1999: S. 45).

30 Vgl. Butler (2001).

31 Foucault (2005: S. 245).

32 Ebd. (S. 246).

33 Ebd.

34 Ebd. (S. 240).

35 Wiede (2014). 
Subjekt, nach Althusser, indem das Individuum eine allgemeine Anrufung, etwa die Ideologien von Staat, Familie etc., als seine eigene annimmt. ${ }^{36}$ Mit Deleuze kann diese Anrufung als initiale Subjektkonstitution und „ständige[r] Verdopplungsprozess“ beschrieben werden, in dem „die äußere Welt - Erfahrungen, Wahrheiten, Normen, Regeln und Vorschriften - nach innen gefaltet und dann wieder entfaltet “37 wird.

Der zentrale Mechanismus der Subjektivierungsmacht ist also die Rückwendung gegen sich selbst, aber zugleich auch ein Bezug auf sich selbst. Es ist dieser inhärente Unterwerfungsakt der Subjektivation, der für die machttheoretische Perspektive nicht nur als disziplinierendes Moment, sondern schließlich auch als produktiver Machteffekt zentral ist. Die Unterwerfung, das Sich-Eingliedern in eine gesellschaftliche, kategoriale Ordnung bringt durch die ermöglichte Subjektwerdung auch einen Autonomieeffekt mit sich, der das Subjekt zu einem handlungsfähigen Akteur macht. Der Althusser'sche Anrufungsakt, darauf weist Butler in Weiterführung Foucaults hin, darf dabei nicht als einzelner Akt der Benennung und als eindeutiger Entstehungsursprung des Subjekts missverstanden werden: Die Anrufung selbst ist Teil einer Verkettung von performativen Akten und Bedeutungsaufführungen, die immer auch deutungsoffen sind. ${ }^{38}$ Die Unterordnung (Subjektivation) ist für Butler deswegen umso stärker auch die Voraussetzung für die politische Formierung und Handlungsfähigkeit des Subjekts. In einem Aneignungsakt des Subjekts gegenüber der unterwerfenden, gründenden, produktiven Macht vollzieht sich eine Statusverschiebung ebendieser Macht. Diejenige Macht, die das Subjekt hervorzubringen vermag, unter das es sich als Bedingung seiner Existenz unterordnen muss, vollzieht sich außerhalb des Subjekts; war ihm vorgängig und konstitutiv wirksam. In einer Umkehrung der Bedingungen seiner Unterordnung nimmt das Subjekt die Macht auf, sodass es in die Lage versetzt wird, diese wiedereinzusetzen und so selbst handlungsfähig zu werden. Dieser produktive Ermächtigungseffekt repräsentiert bei Butler die gewollte Unterordnung des Subjekts unter die Subjektivierungsmacht sowie gleichzeitig den Widerstand des Subjekts dagegen. Er besteht darin, dass die übernommene Macht gegen jene Macht arbeiten kann, die die Übernahme erst ermöglicht hat. Subjektivierungsmacht besteht demnach für Butler in der grundlegenden Abhängigkeit von äußeren diskursiven Machtverhältnissen, denen sich das Individuum gezwungenermaßen aus

36 Vgl. Althusser (2010: S. 88 f.).

37 Deleuze (1988), zitiert nach Sauter (2014: S. 36).

38 Vgl. Butler (2001). 
einem Existenztrieb heraus unterwirft, jedoch paradoxerweise dadurch erst seine Handlungsfähigkeit erhält. Trotz des Zwangs der Unterordnung hat Subjektivierungsmacht aus dieser Perspektive eine unverkennbar produktive und konstruktive Seite, in dem Sinne, dass Handlungsfähigkeit als Wirkung von Unterordnung verstanden wird.

\section{Digitale Subjektivierungsmacht als Technologie des Selbst}

Die Aufsätze von Hannelore Bublitz ${ }^{39}$, Tanja Paulitz ${ }^{40}$ und Theresa Sauter ${ }^{41}$ gehören zu den wenigen und aufschlussreichen theoretischen Auseinandersetzungen mit digitaler Subjektivierungsmacht. ${ }^{42}$ Hier werden Subjekte weniger statisch, sondern als dynamisch und veränderbar verstanden; ${ }^{43}$ als Entitäten, die sich in ständig wiederholenden, performativen Selbst-Werdungsprozessen in Bezug auf ein Außen erzeugen. Die digitale Subjektivität wird ebenso wie die Offline-Subjektivität ${ }^{44}$ als eine „sich konstant verändernde Form, die durch wechselseitige Beziehungen zu sich selbst und zu anderen geformt und überarbeitet wird und von Kontext zu Kontext variiert" 45 , konzipiert.

Digitale Subjektivierung wird bei Bublitz und Paulitz mit der Foucault'schen Denkfigur der Technologie des Selbst analysiert. Die Funktionsweise der Selbst-Technologie ist dadurch bestimmt, dass die gesellschaftliche Selbstwerdung über Disziplinierung und Normalisierung von Körper und Seele verläuft, dabei nicht allein durch ein Regieren von außen entsteht, sondern durch ein Sich-Selbst-Regieren der sich der Gesellschaft unterwerfenden Individuen. ${ }^{46}$ Bublitz hebt hervor, dass sich das Individuum in sozialen Netzwerken zunehmend selbst „als soziales Subjekt aus und

39 Vgl. Bublitz (2014).

40 Vgl. Paulitz (2014).

41 Vgl. Sauter (2014).

42 Für weitere empirische Auseinandersetzungen siehe auch Carstensen (2014); Siri (2014).

43 Vgl. Sauter (2014).

44 Die Bezeichnung, Offline-Subjekt' ist insofern problematisch, als dass damit ein ,wirkliches' Selbst als Negation in Bezug auf ein bedeutungsvolleres virtuelles Selbst definiert zu sein scheint. Aus Gründen der Übersichtlichkeit der Argumentationsführung soll im Folgenden trotzdem an dieser dualistischen und subtil hierarchisierenden Gegenüberstellung festgehalten werden, ohne jedoch diese Wertung zu suggerieren.

45 Sauter (2014: S. 26).

46 Vgl. Bublitz (2014); Sauter (2014). 
her[stellt; Anm. J.V.S. ]“47. Dies geschehe durch die Bezugnahme auf und Einordnung in eine soziale Umwelt und diskursive, gesellschaftliche Strukturen, in die das Individuum eingebettet ist. Im Kontext des Web 2.0, wie auch außerhalb dessen, wird das Individuum „durch reflexive Wendung, von der Anrufung zurück auf sich selbst [...] zu einem Selbst, das sich in Selbstexploration, Selbstmodellierung und Selbstexpression als Objekt seiner selbst konstituiert, ein Bild von sich entwirft und sich eine eigene Gestalt gibt" ${ }^{\text {48 }}$. Die digitale Subjektivierungsmacht zeichnet sich dabei vor allem durch medial vermittelte und ermöglichte Selbststeuerungs- und Erzeugungsmechanismen aus, die auch „über Rekursion und Feedbackschleifen "49 den Akt der Unterwerfung, Einordnung, (Selbst-)Adjustierung und der Orientierung des Selbst am Außen vollziehen.

Neben der Betonung der aktiven Einbindung des Subjekts in seine medienbasierte Selbstproduktion wird auch die Unterwerfung durch Normalisierungsdruck nicht mehr allein als Existenzvoraussetzung und Orientierung gesehen, sondern auch als eine Individualisierung und Abgrenzungspraxis verstanden: „An die Stelle der Ausrichtung an vorgegebene soziale Normen und Pflichten treten dynamische Orientierungsmuster; Subjektivierung zielt jetzt auf flexible Selbstkonstruktionen ${ }^{\text {50 }}$. Angelehnt an die Normalismustheorie von Jürgen Link ${ }^{51}$ beschreibt Bublitz das Normalitätsspektrum in sozialen Netzwerken nicht als fixe Norm, sondern als eine anschlussfähige Differenz. Der flexible Normalismus beinhalte nach wie vor eine Ausrichtung der Subjekte an externen Normen und Zwängen, orientiere sich jedoch auch an der Richtlinie individueller Differenzierung. ${ }^{52}$ Digitale Subjektivierung richtet sich, so Bublitz, weniger an Normalität als an der Individualisierung des Online-Subjekts aus. ${ }^{53}$ Die Rede von Normalisierungstendenzen in sozialen Netzwerken ist aus dieser Sicht insofern missverständlich, als bestimmte und besondere Eigenschaften des Individuums/Subjekts hervorgehoben und vom Allgemeinen abgegrenzt werden. Das durch soziale Netzwerke selbst dynamisch und interaktiv gewordene Vergleichsfeld fordere eine „flexible Selbst[er]findung“ ein. ${ }^{54}$ Die sozialen Netzwerke werden deswegen nicht nur als Foren betrachtet, auf de-

47 Bublitz (2014: S. 12).

48 Bröckling (2007), zitiert nach Bublitz (2014: S. 17).

49 Bublitz (2014: S. 17).

50 Ebd. (S. 9).

51 Vgl. Link (1997).

52 Vgl. ebd.; Bublitz (2014).

53 Vgl. Bublitz (2014).

54 Ebd. (S. 16). 
nen die altbekannte Anpassung an gesellschaftlich vorgegebene Anforderungen vollzogen wird. Obwohl das digitale Subjekt als „participatory subject “ ${ }^{55}$ an Selbst-Darstellung und -Veröffentlichung existentiell gebunden und aktiv beteiligt ist, um nicht in Bedeutungslosigkeit zu verschwinden, ${ }^{56}$ werden die hochdynamischen „medial inszenierte[n] Bericht- und Geständnispraktiken " 57 als eine produktive und bisweilen sogar konstruktiv ermächtigende, sprachliche und körperliche Selbst-Demonstration des Subjekts aufgefasst. Bublitz, Sauter und Paulitz betonen in diesem Sinne die emanzipatorische Pointe der Butler'schen Subjektivierungstheorie, indem sie digitale Subjektivierung als produktive, potentiell ermächtigende Machtform begreifen. ${ }^{58}$ Mit dieser tendenziell sozial-deterministischen Deutung werden digitale Subjektivierungsprozesse und die sogenannte Selbstoffenbarung weniger als „erzwungene Praxis im Dienste einer Ordnungsmacht" ${ }^{* 59}$, denn als „performative Produktion des Subjekts innerhalb öffentlicher Konventionen " 60 gedeutet. Diese wiederum werde durch digitale Medientechnologien zunehmend aufgelockert und wirke schwächer normalisierend.

Der Fokus auf die Bildung des Subjekts und die Zusammenführung produktiver und einschränkender Auswirkungen seiner Machtunterordnung als seine Daseinsbedingung ist wichtig, um vom Verständnis des liberalen Subjekts hin zu einem Subjektbild zu gelangen, das grundsätzlich gesellschaftlich, aber nicht vollends determiniert und fremdbestimmt gedacht ist. Doch kann die produktive Pointe der Subjektivation Gefahr laufen, die Ambivalenzen der Subjektivierungsmachttheorie schließlich doch auf eine Seite hin aufzulösen und dadurch die Machtdurchdrungenheit des Subjekts zu harmonisieren. Trotz der Butler'schen Wendung, sollte der Aspekt der Subjektivation vor allem im Digitalen nicht primär emanzipatorisch gelesen werden. Die Technologie des Selbst bleibt eine Form von Regierungspraxis. Weil, wie Foucault erkennt, Führung zur Selbst- und Fremdführung zentrales Merkmal von gegenwärtiger Regierungsrationalität ist, sollte auch Digitale Subjektivierungsmacht als Verstrickung der Re-

55 Bucher (2012a: S. 1175). Partizipation wird als Norm in sozialen Netzwerken gefördert, da das aktive, kommunizierende und interagierende Subjekt für die Plattformbetreiber am nützlichsten für den traffic und schließlich am profitabelsten ist. Vgl. auch Bublitz (2014); Sauter (2014).

56 Vgl. Muhle (2018).

57 Bublitz (2014: S. 12).

58 Vgl. ebd.; Paulitz (2014); Sauter (2014).

59 Butler (2003: S. 119), zitiert nach Bublitz (2014: S. 12).

60 Ebd. 
gierung des Selbst und des Fremd-Regierens theoretisiert werden. Der Beitrag der Subjektivierungsmachttheorie Foucaults ist es gerade, den Subjektivierungsprozess vor allem machttheoretisch zu begreifen und dadurch die Ambivalenz der Subjektivierung zu betonen. Es sollte deutlich werden, dass Subjektivierung durch eine Verwicklung von repressiven und produktiven Dynamiken geprägt ist. Die Anerkennung dieser Zweischneidigkeit ist für eine gesellschaftstheoretisch sensible Sichtweise zentral; die Überbetonung der produktiven Macht in digitalen Subjektivierungszusammenhängen verkennt die systematischen, apparativen Zusammenhänge, durch die Herrschaftsdynamiken und intervenierende Regierungsrationalitäten sichtbar werden können.

\section{Digitale Subjektivierungsmacht als Digitales Selbst}

Um die machttheoretische Problematik digitaler Subjektivierung hervorzuheben, soll nun mit dem Konzept des Digitalen Selbst der Aspekt der repressiven, techno-sozial konstituierten Regierung von Außen in der digitalen Subjektivierung aufgegriffen werden. Der aus dem Privatheitsdiskurs entliehene, nicht explizit als Subjektivierungsmachttheorie entwickelte Ansatz des Digitalen Selbst ${ }^{61}$ nach John Cheney-Lippold ist hilfreich, um digitale Subjektivierungsmacht um eine herrschaftskritische und medientheoretische Perspektive zu ergänzen.

Mit dem Konzept des Digitalen Selbst kann neben der privatheitsgefährdenden Praxis der digitalen Selbstveröffentlichung die Ebene der digitalen Infrastrukturen in den Vordergrund gerückt werden. Dieses Subjektivierungsphänomen beschreibt die systematisierte digitale Datenansammlung, die einer realen Person zugeordnet werden und als ihr Abbild in der virtuellen Welt gelten kann. ${ }^{62}$ Basierend auf Informationen getrackter Netzaktivitäten durch Profiling-Methoden ${ }^{63}$ und Meta-Daten wird auf Eigenschaften, Identitätsmerkmale oder auch psychologische Muster von Offline-Subjekten geschlossen, d. h. es werden entsprechende Zuschreibungen für das digitale Subjekt erzeugt. ${ }^{64}$ Durch diese sogenannten Personalisierungstech-

61 Auch data self oder algorithmische Identität, vgl. Cheney-Lippold (2011); vgl. auch das Konzept des Dividual bei Deleuze (1992).

62 Vgl. Graham/Wood (2003).

63 Z. B. geotargeting, demographisches targeting, behavioural targeting und predictive behavioural targeting, vgl. Becker (2013).

64 Vgl. Kosinski et al. (2013); Muhle (2018). 
nologien ${ }^{65}$ über Algorithmen und computergestützte Systeme wird das Offline-Subjekt als Datendarstellung vermeintlich repräsentiert. Die Repräsentation wird so von unserem Selbst getrennt (es wird nach Deleuze zum Dividuum $^{66}$ ) und das Subjekt auf Informationen und Ergebnisse von Berechnungen reduziert. Cheney-Lippold beschreibt das Digitale Selbst als „new algorithmic identity“ ${ }^{67}$ die aus der systematischen Zusammenführung von Identitätskategorien auf der Basis des algorithmisch verarbeiteten Internetverhaltens von Subjekten bestehe:

The networked infrastructure of the internet, with its technological capacity to track user movements across different web sites and servers, has given rise to an industry of web analytics firms that are actively amassing information on individuals and fine-tuning computer algorithms to make sense of that data. The product of many of these firms is a 'new algorithmic identity', an identity formation that works through mathematical algorithms to infer categories of identity on otherwise anonymous beings. ${ }^{68}$

Allerdings scheinen das Konzept und die Kritik des Digitalen Selbst teilweise auf ein liberales Subjektverständnis zu verweisen, in dem kategoriale Identität nicht mit Macht/Wissen verwickelt ist, Selbstkontrolle keine Regierungsform darstellt und in der die Bedeutung des Selbst als keine grundlegend gesellschaftliche Ausdrucksweise gesehen wird. In der Privatheitstheorie steht das Konzept des Digitalen Selbst für das transparent gemachte Selbst einerseits und das Ausgeliefertsein des Subjekts gegenüber den gesellschaftlichen, digitalen Infrastrukturen andererseits. Dabei wird das neue, digitale, datenbasierte Selbst auf Grund seiner normalisierenden, disziplinierenden und datengesteuerten Konstitution dem Offline-Subjekt gegenübergestellt, das somit als repräsentierbar, frei und uneingeschränkt handlungsfähig erscheint. Diese Suggestion wird teilweise sogar explizit gemacht, wie zum Beispiel bei Jennifer R. Whitson: „Instead of individuals - irreducible and with an autonomous sense of agency - the new subject of governance is $[\ldots]$ an artifact of data mining searches and computer pro-

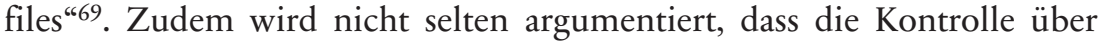
Identitätskategorien verloren werde und dass eine solche Kontrolle analog zu Autorschaft oder Eigentum funktioniere. Wie auch bei Cheney-Lippold

65 Vgl. Negroponte (1995).

66 Vgl. Deleuze (1992).

67 Cheney-Lippold (2011: S. 165).

68 Ebd. (Herv. i. Orig.).

69 Whitson (2014: S. 343). 
dargelegt, ist das Offline-Subjekt nicht nur seiner Selbst bewusst, sondern sich selbst gestaltend und besitzend: „We are effectively losing control in defining who we are online, or more specifically we are losing ownership over the meaning of the categories that constitute out identities"70. Als besondere Eigenschaft des Digitalen Selbst wird hervorgehoben, dass es nicht, oder zumindest nur indirekt über das Zutun des Subjekts entstehe,

because the logic, maintenance, and redesign of these algorithms remain in the hands of the information providers, they are in a distinctly privileged position to rewrite our understanding of them, or to engender a lingering uncertainty about their criteria that makes it difficult for us to treat them as truly our own. ${ }^{71}$

Während die Unsichtbarkeit der (Re-)Produktion des Digitalen Selbst auf ein Transparenzproblem verweist, wird oft implizit vermittelt, ,normale Subjektivierung sei ein Ergebnis autonomer Praxis und reflektierter Entscheidungen und vollziehe sich durch freie Gestaltungsmöglichkeiten. In Anbetracht der oben erläuterten, notwendigen Unterwerfung des Subjekts, um seine Entstehung und sein Fortbestehen zu garantieren, und in Anbetracht der Gesellschaftlichkeit von Identitätskategorien, ist eine solche privatheitstheoretische Kritik am Digitalen Selbst verkürzt. Dagegen will ich für eine Lesart plädieren, in der das Digitale Selbst weder auf Grund seiner vermeintlichen Eigenschaften noch wegen seines fremdbestimmten Entstehungs- und Entwicklungsprozesses grundlegend mit dem Offline-Subjekt kontrastiert wird. Denn mit den Erkenntnissen der poststrukturalistischen Subjektivierungstheorie unterscheidet sich digitale Subjektivierung weder durch die Existenz gesellschaftlichen Einflusses noch durch das prinzipielle Wirken von Macht durch Unterwerfungsmechanismen von Offline-Subjektivierungsprozessen. Stattdessen, so will ich hervorheben, sind es die technische Verwicklung und Einbettung in den digitalen Kapitalismus und den digitalen Staat, die eine Differenzierung der Subjektivierungsweisen und von Subjektivierungsmacht möglich und notwendig machen. Subjektivation im Digitalen ist auf Grund der Nutzung und Funktionsweisen digitaler Infrastruktur und algorithmischer Techniken durch andere Unterwerfungsmechanismen und Machtdynamiken geprägt - eine Differenzierung, die das Konzept des Digitalen Selbst, trotz seiner teilweise liberalen Engführungen, durch seine medientheoretische Sensibilität hervorheben kann.

70 Cheney-Lippold (2011: S. 178).

71 Gillespie (2014: S. 21). 
Der von Paulitz und Bublitz mobilisierte Ansatz der digitalen Technologie des Selbst ist wegen seiner Betonung des Semantischen nicht ausreichend, um digitale Subjektivierungsmacht in ihrer Gesamtheit und in ihrer politischen Relevanz zu greifen. Weil der Fokus dieser post-strukturalistisch inspirierten Analysen auf der kulturellen Ebene, der Ebene der sprachlichen und symbolischen Ausdrucksformen und der inhaltlichen (Selbst-)Darstellung des Subjekts liegt, werden Unterordnungsmechanismen im Subjektivierungsprozess vor allem durch diskursive Konstellationen aufgezeigt. Somit wird aus dieser Perspektive das Phänomen der digitalen Subjektivierungsmacht als Wiederholung oder Spiegelung der Offline-Subjektivation theoretisiert. Problematisch daran ist, dass der diskursive Blick auf digitale Subjektivierungsprozesse suggeriert, dass Medientechnologien vor allem instrumentell seien und ein optimal an ihre Zwecke anzupassendes Mittel darstellten. ${ }^{72} \mathrm{Um}$ die medial verschobene Subjektivierungsdynamik aufzuzeigen, ist es demgegenüber notwendig, der Subjektivierung auf der Ebene der Frontend-Prozesse (der Perspektive auf digitale Subjektivierungsmacht als Technologie des Selbst) eine Perspektive hinzuzufügen, die das Backend der Medientechnologien in die Machtanalyse miteinbezieht. Durch die medientheoretische Beleuchtung digitaler Subjektivierung können gegenüber der produktiven Dynamik Objektivierungsaspekte der digitalen Subjektivation hervorgehoben und dabei schließlich repressive Machtelemente und Herrschaftsstrukturen kenntlich gemacht werden.

Mit dem Konzept des Digitalen Selbst kann gezeigt werden, dass das zentrale Alleinstellungsmerkmal digitaler Subjektivation nicht eine intersubjektive, gesellschaftliche, sondern auch - und vor allem - eine datenbasierte, algorithmische Konstruktion des digitalen Subjekts ist. Dieser Subjektivierungsprozess wird nicht nur durch die digitale Infrastruktur vermittelt, sondern auch durch sie konstituiert: Offline-Subjekte entstehen nur bedingt durch eine komplexe Verwicklung ortsgebundener, sozialer Assoziationen und Interaktionen. Denn es sind ebendiese Prozesse, die durch Daten und Software bestimmt und nicht ohne sozio-technisch in Kraft gesetzte Berechnungslogik verstanden werden können. Durch algorithmische Profilbildung oder kybernetische Kategorisierung ${ }^{73}$ und nicht allein durch semiotische und semantische Selbstkonstruktion entsteht das digitale Subjekt, das sich einer berechnenden Subjektivierungsmacht unterwirft:

72 Vgl. McLuhan (1964).

73 Vgl. Cheney-Lippold (2011). 
[B] uried underneath the signifying surfaces of the computer interface, embodied in abstract protocols, written in calculable documents and wired materials, software [Herv. J.V.S.] engages in processes of subjectivation. $^{74}$

Im Web 2.0 wird eine „computer-aided subjectivity“75 produziert. Die Unterwerfung findet nicht unter diskursive Kategorien und diffuse gesellschaftliche Normenkonstellationen statt. Vielmehr sind es Bits und Bytes, exakte rechnerische, algorithmische Prozesse, die das kategoriale und schließlich normative Bezugssystem konstituieren, von dem Macht in den digitalen Subjektivierungsprozessen ausgeht. Durch die algorithmusgesteuerte Auswertung von Daten und die Erzeugung von Profilen sowie deren Systematisierung entsteht eine andersartige Form der Subjektivation, die das Objektivierungsmoment des Subjektivierungsprozesses intensiviert. Objektivierung findet entlang operativer Rechenlogiken statt; sprachlich-normative Wege der Klassifikation werden durch algorithmisch-faktische ergänzt. ${ }^{76}$ Die digitale Subjektivation funktioniert damit nicht allein über eine normativ-diskursive Anrufung und einen abstrakten Referenzrahmen, sondern vor allem durch - wenn auch normativ durchdrungene - automatisierte Berechnungslogiken und algorithmische Rechenprozesse, die Daten verarbeiten, auswählen, bewerten, sortieren und somit ,Subjekte‘ ohne expliziten Anrufungsmechanismus formen.

Das Außen, auf das sich das Individuum in seiner Selbstkonstitution im Subjektivierungsprozess aktiv bezieht, ist dabei nicht vorrangig als komplexe lokale Machtkonstellation zu verstehen, sondern stellt vielmehr eine materialisierte und technisierte kapitalistische Herrschaftskonstellation dar. Die algorithmische Gestaltung der digitalen Infrastruktur durch globale Akteure des Kapitalismus und deren Kooperation mit dem überwachenden, kontrollierenden, strafenden Staat ${ }^{77}$ sind ausschlaggebend für die Funktionsweise des Digitalen Selbst: ${ }^{78}$

Subjektivierung [vollzieht; Anm. J.V.S.] sich gegenwärtig [...] nicht mehr in institutionalisierten Einschließungsmilieus, sondern schließt

74 Bucher (2012b: S. 481).

75 Guattari (1989: S. 133).

76 Vgl. Hörtnagel (2019).

77 Vgl. Lyon (2014).

78 Wie auch Bublitz (2014) anerkennt, indem sie das Dispositiv des medialen Beichtstuhls kapitalismuskritisch deutet. 
sich unmittelbar mit der Dynamik eines globalisierten Konsumkapitalismus und globaler Medientechnologien zusammen. ${ }^{79}$

Durch die Materialisierung in Rechenprozessen tritt diese Machtkonstellation besonders verfestigt als Herrschaft auf. Herrschaft bezeichnet eine Sondererscheinung von Machtbeziehungen: während letztere lokal und dynamisch sind, sind Herrschaftszustände dauerhaft und wenig beweglich. ${ }^{80}$ Herrschaft ist in diesem Sinne „eine strategische Machtstruktur, deren Bedeutung und Folgen bis in die kleinste Verästelung der Gesellschaft reichen" und die „über lange geschichtliche Zeiträume herausgebildet und verfestigt" wurde. ${ }^{81}$ Als Herrschaft verdichtet Macht sich so, dass sie zu einer institutionalisierten Struktur wird, bei der eine systematische Anordnung der Machtbeziehungen ausfindig zu machen ist. Durch die Linse des Konzepts des Digitalen Selbst stellt sich digitale Subjektivierungsmacht damit weniger als eine produktive, dynamische Form von Machtbeziehungen dar, als vielmehr eine, die statisch ist und im klassischen Sinne repressiv wirkt. Denn die personenbezogenen Datensammlungen und Sinnzuschreibungen werden, so die spätestens durch Edward Snowden belegte Diagnose, nicht nur für den kommerziellen Zweck, sondern auch für polizeiliche oder geheimdienstliche Zwecke verwendet. ${ }^{82}$ Das Digitale Selbst prägt folglich dauerhaft die analogen Möglichkeitsräume des Offline-Subjekts: „Comprehensive computerization samples and assembles reality in a variety of ways and thus shapes the forms of perceiving and acting upon

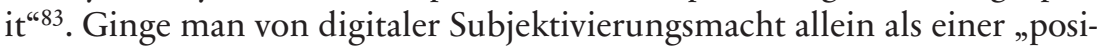
tive[n] Lebensmacht" ${ }^{\text {84 }}$ aus, die über die Technologie des Selbst regulierende, aber produktive Macht ausüben kann, würde man verkennen, dass sich die Subjektivierungsweise im digitalen Kontext verschoben hat und die algorithmischen Subjektivierungsprozesse des Digitalen Selbst im Backend nicht als produktive Machteffekte verstanden werden können, sondern im Gegenteil: sie die Ausgesetztheit des Subjekts gegenüber bestehenden Herrschaftszuständen intensivieren.

79 Bublitz (2014: S. 9).

80 Vgl. Foucault (2005).

81 Foucault (1989: S. 115).

82 Vgl. Lyon (2008).

83 Kallinikos (2010: S. 2).

84 Foucault (1989: S. 166). 


\section{Fazit: Zweifache Subjektivierungsmacht}

Durch beide Perspektiven, die in Kombination das Zusammendenken beider Pointen der Subjektivierungsmacht ermöglichen, lässt sich digitale Subjektivierungsmacht differenziert analysieren. Beide Subjektivierungsmachtdynamiken - das Digitale Selbst und die digitale Technologie des Selbst -, dies hat die post-strukturalistische Machttheorie gezeigt, wirken objektivierend und sind nicht primär durch Selbstbestimmung geprägt. Eine differenzierte Betrachtung durch die Zusammenführung von post-strukturalistischen und privathheitstheoretischen Ansätzen kann, ohne auf liberale Subjektkonzeptionen zurückzufallen und ohne die spezifische Medienanalyse zu ignorieren, die besondere Form der Macht und der Objektivierung in digitalen Subjektivierungsprozessen aufzeigen. Indem ich die Konzepte des Digitalen Selbst und der digitalen Technologie des Selbst für eine gegenseitige Kritik fruchtbar gemacht habe, habe ich mit dem parallelen Lesen zwei Thesen stark zu machen versucht: Erstens sensibilisiert die Perspektive post-strukturalistischer Machttheorien nach Althusser, Foucault und Butler für Machtmechanismen im Subjektivierungsprozess, indem sie aufzeigt, dass Macht für das Subjekt konstitutiv ist, und es selbst in seiner Konstituierung bereits dem Gesellschaftlichen unterworfen ist, bevor es erst als potentiell Macht ausübendes Subjekt handlungsfähig werden kann. Über Foucaults methodischen Zugang - Macht als Gefüge produktiver, lokaler Machtbeziehungen zu analysieren - geraten in den Weiterentwicklungen zu Ansätzen post-strukturalistischer, digitaler Subjektivierungstheorie jedoch zweitens die gegenwärtig in digitalen Subjektivierungsprozessen wirksamen medientechnologischen Strukturen und die kapitalistische Verwertung der performativen Praxis der digitalen Selbstproduktion aus dem Blickfeld. Während Foucault noch bemüht ist, die „eigentliche Verankerung der Macht [...] außerhalb der Institutionen zu suchen" ${ }^{* 85}$, zeigt die Analyse der algorithmischen Subjektivierungsmacht durch das Konzept des Digitalen Selbst, dass sie systematischen Charakter hat und in technologischen Strukturen nicht nur Gestalt annimmt, sondern durch sie eine neue Form erhält.

Ich habe mit der zweifachen Interpretation von Subjektivierungsmacht versucht zu zeigen, dass der Unterwerfungsprozess als Bedingung der Handlungsfähigkeit des Subjekts im Digitalen und als Machtaneignung nicht überschätzt werden darf. Es muss vielmehr festgehalten werden, dass, wie Carsten Ochs und Barbara Büttner aus ihrer empirischen Studie

85 Foucault (2005: S. 258). 
zu Quantified-Self-Technologien schließen, „Selbstbestimmung und die Bestimmung des Selbst [...], daten-ökonomisch getrieben, auseinanderzulaufen [scheinen; Anm. J.V.S.]"86. Ausgangspunkt dieser Überlegung ist nicht das autonome Subjekt, sondern das gesellschaftlich abhängige Subjekt, das sich Selbstbestimmung durch Unterordnung ermöglichen kann. Je mehr Sichtbarkeit erzeugt und gesellschaftliche Einordnung vollzogen wird, desto gestärkter ist die soziale Existenz und dadurch die potentielle Ermächtigung. Im Zuge der gleichen Prozesse wird aber auch der Herrschaftsmechanismus der digitalen Subjektivation betätigt: Die Technologie des Selbst als aktive Selbstkonstruktion in sozialen Netzwerken bildet die Voraussetzung für die repressiven Effekte der überwachungskapitalistischen Datenökonomie. ${ }^{87}$ Die digitale Infrastruktur intensiviert die Unterwerfungsdynamik im Online-Subjektivierungsprozess, indem „die datenökonomische Monetarisierung [...] die Übersetzung von Subjektivität in Objektivierungspotentiale, und die ökonomische Nutzbarmachung letzterer [ermöglicht; Anm. J.V.S.] ${ }^{\text {(88 }}$. Hierbei vollzieht sich eine Verdrehung der produktiven Pointe der Subjektivierungsmachttheorie: Während im Web 2.0 potentiell Ermächtigungseffekte und Handlungspotentiale durch das Akzeptieren von Anrufungen und die Reproduktion gesellschaftlicher Kategorien in der Subjektkonstituierung entstehen, laufen gleichzeitig algorithmische Objektivierungsprozesse im Backend ab, die wiederum die Handlungspotentiale einschränken können. Und mehr noch: „[J]e datenintensiver [...] am Front-End Subjektivierungspraktiken [...] [vollzogen werden; Anm. J.V.S.], desto tiefenschärfer wird sich ihre Objektivierung am Back-End gestalten" ${ }^{89}$. Durch Objektivierungsmechanismen erlangte Ermächtigungseffekte bedeuten online also immer auch eine Intensivierung der datengesteuerten Objektivierung. Der ermächtigenden Technologie des Selbst wird durch die algorithmische Konstruktion des Digitalen Selbst mindestens entgegengewirkt. Obwohl Subjekte prinzipiell durch gesellschaftliche Unterordnung, Selbstadjustierung und Selbstoptimierung hervorgebracht werden, ist die dynamische Bewegung der Subjektivierungsmacht, in der sich das Subjekt die Macht anschließend aneignet, eingeschränkt. Denn Subjektivierungsmacht im Internet ist immer auch mathematisiert; die Übersetzung ins Digitale Selbst, die Produktion von Datensubjekten, ist demnach besonders objektivierend.

86 Ochs/Büttner (2019: S. 210).

87 Vgl. Bublitz (2014).

88 Ochs/Büttner (2019: S. 210).

89 Ebd. 
Mit der Zusammenführung vom Digitalen Selbst, digitaler Technologien des Selbst und der Anerkennung ihrer Ambivalenzen kann die Verknüpfung von produktiven und repressiven Machtformen sowie das Wechselspiel von Fremd- und Selbstkontrolle in digitalen Subjektivierungsprozessen betont und herausgearbeitet werden. Dies ist eine wichtige Grundlage, um in weiteren Schritten Lösungsansätze von Überwachungs- und Privatheitsproblematiken zu diskutieren und Einschränkungen durch Fremdbestimmung sowie Möglichkeiten der Selbstkontrolle macht- und medientheoretisch informiert einzubeziehen. Die spezifischen Herrschaftsmomente digitaler Subjektkonstruktion sowie die existenzielle Unterwerfung des Subjekts müssen gleichzeitig als eine Form der zweifachen Unterwerfung anerkannt werden, um schließlich einen subjekttheoretischen, gesellschaftskritischen Umgang mit digitaler Überwachung im Web 2.0 und mit individualisiertem Privatheitsmanagement zu ermöglichen.

\section{Literaturverzeichnis}

Althusser, Louis (2010): Ideologie und ideologische Staatsapparate. Hamburg: VSA.

Becker, Jörg (2013): Die Digitalisierung von Medien und Kultur. Wiesbaden: Springer VS.

Becker, Konrad/Stalder, Felix (2009): Deep Search. The Politics of Search beyond Google. New Jersey: Transaction Publishers.

Bennett, Colin J. (2011): „In defence of privacy: The concept and the regime“. In: Surveillance \& Society 8 (4), S. 485-496.

Bröckling, Ulrich (2007): Das unternehmerische Selbst. Soziologie einer Subjektivierungsform. Frankfurt am Main: Suhrkamp.

Bublitz, Hannelore (2014): „Im Beichtstuhl der Medien - Konstitution des Subjekts im öffentlichen Bekenntnis“. In: Paulitz, Tanja/Carstensen, Tanja (Hrsg.): Subjektivierung 2.0. Machtverhältnisse digitaler Öffentlichkeiten. Wiesbaden: Springer VS, S. 7-22.

Bucher, Taina (2012a): „Want to be on the top? Algorithmic power and the threat of invisibility on Facebook“. In: New Media \& Society 14 (7), S. 1164-1180.

Bucher, Taina (2012b): „The Friendship Assemblage: Investigating Programmed Sociality on Facebook“. In: Television \& New Media 14 (6), S. 479-493.

Butler, Judith (2001): Psyche der Macht. Das Subjekt der Unterwerfung. Frankfurt am Main: Suhrkamp.

Butler, Judith (2003): Kritik der ethischen Gewalt. Frankfurt am Main: Suhrkamp. 
Carstensen, Tanja (2014): „Öffentliche Selbstdarstellung im Internet als Aufwand. Digitale Subjektwerdung am Beispiel von jungen Menschen in Internetberufen“. In: Paulitz, Tanja/Carstensen, Tanja (Hrsg.): Subjektivierung 2.0. Machtverhältnisse digitaler Öffentlichkeiten. Wiesbaden: Springer VS, S. 83-100.

Cheney-Lippold, John (2011): „A New Algorithmic Identity: Soft Biopolitics and the Modulation of Control“. In: Theory, Culture \& Society 28 (6), S. 164-181.

Cohen, Julie E. (2012): Configuring the Networked Self. New Haven: Yale University Press.

Couldry, Nick/Hepp, Andreas (2016): The mediated construction of reality. London: Polity Press.

DeCew, Judith Wagner (2015): „The feminist critique of privacy: past arguments and new social understandings“. In: Rössler, Beate/Mokrosinska, Dorota (2015): Social Dimensions of Privacy. Interdisciplinary Perspectives. Cambridge: Cambridge University Press, S. 85-103.

Deleuze, Gilles (1988): Foucault. Minnesota: University of Minnesota Press.

Deleuze, Gilles (1992): „Postscript on the Societies of Control“. In: October 59, S. 3-7.

Dourish, Paul/Palen, Leysia (2003): „Unpacking ,privacy“ for a networked world“. In: CHI '03: Proceedings of the SIGCHI Conference on Human Factors in Computing Systems. URL: https:/www.dourish.com/publications/2003/chi2003-priv acy.pdf [Abruf am: 21.09.2019].

Foucault, Michel (1982): „The Subject and Power“. In: Critical Inquiry 8 (4), S. 777-795.

Foucault, Michel (1989): Der Wille zum Wissen. Frankfurt am Main: Suhrkamp.

Foucault, Michel (1999): Beyond Structuralism and Hermeneutics. Chicago: Chicago University Press.

Foucault, Michel (2005): Analytik der Macht. Frankfurt am Main: Suhrkamp.

Gentzel, Peter et al. (2019): „Einführung: Das vergessene Subjekt in der Kommunikationswissenschaft“. In: Dies. (Hrsg.): Das vergessene Subjekt. Subjektkonstitutionen in mediatisierten Alltagswelten. Wiesbaden: Springer VS, S. 1-14.

Gillespie, Tarleton (2014): „The Relevance of Algorithms“. In: Gillespie, Tarleton et al. (Hrsg.): Media Technologies: Essays on Communication, Materiality, and Society. Cambridge: MIT Press, S. 167-197.

Guattari, Félix (1989): „The Three Ecologies“. In: New Formations 8, S. 131-147.

Hörtnagl, Jakob (2019): „Subjektivierung in datafizierten Gesellschaften - Dividualisierung als Perspektive auf kommunikative Aushandlungsprozesse in datengetriebenen Zeiten“. In: Gentzel, Peter et al. (Hrsg.): Das vergessene Subjekt. Subjektkonstitutionen in mediatisierten Alltagswelten. Wiesbaden: Springer VS, S. 135-158.

Kosinski, Michal et al. (2013): „Private traits and attributes are predictable from digital records of human behavior". In: Proceedings of the National Academy of Sciences of the United States of America (PNAS) 110 (15), S. 5802-5805. 
Link, Jürgen (1997): Versuch über den Normalismus. Wie Normalität produziert wird. Opladen: Westdeutscher Verlag.

Lyon, David (2014): „Surveillance, Snowden, and Big Data: Capacities, consequences, critique“. In: Big Data \& Society 1 (2), S. 1-13.

McLuhan, Marshall (1964): Understanding Media: The Extensions of Man. Cambridge: MIT Press.

Muhle, Florian (2018): „Stochastically modelling the user: Systemtheoretische Überlegungen zur ,Personalisierung' der Werbekommunikation durch Algorithmen“. In: Mämecke, Torben et al. (Hrsg.): Bedeutende Daten. Modelle, Verfahren und Praxis der Vermessung und Verdatung im Netz. Medien - Kultur Kommunikation. Wiesbaden: Springer VS, S. 143-170.

Negroponte, Nicholas (1995): Being Digital. New York: Vintage Books.

Nissenbaum, Helen (2010): Privacy in Context. Technology, Policy, and the Integrity of Social Life. Stanford: Stanford University Press.

Ochs, Carsten /Büttner, Barbara (2019): „Selbstbestimmte Selbst-Bestimmung? Wie digitale Subjektivierungspraktiken objektivierte Datensubjekte hervorbringen“. In: Ochs, Carsten et al. (Hrsg.): Die Zukunft der Datenökonomie. Zwischen Geschäftsmodell, Kollektivgut und Verbraucherschutz. Wiesbaden: Springer VS, S. 181-214.

Paulitz, Tanja (2014): „Subjektivierung und soziale Praxis im Kontext des Web 2.0“. In: Paulitz, Tanja/Carstensen, Tanja (Hrsg.): Subjektivierung 2.0. Machtverhältnisse digitaler Öffentlichkeiten. Wiesbaden: Springer VS, S. 1-6.

Reckwitz, Andreas (2008): Subjekt. Bielefeld: transcript.

Reigeluth, Tyler (2015): „Warum ,Daten“ nicht genügen. Digitale Spuren als Kontrolle des Selbst und als Selbstkontrolle“. In: Zeitschrift für Medienwissenschaft 13 (7), S. 21-34.

Sauter, Theresa (2014): „Öffentlichmachung privater Subjekte im Web 2.0: Eine Genealogie des Schreibens als Selbstkritik“. In: Paulitz, Tanja/Carstensen, Tanja (Hrsg.): Subjektivierung 2.0. Machtverhältnisse digitaler Öffentlichkeiten. Wiesbaden: Springer VS, S. 23-40.

Siri, Jasmin (2014): „privat*öffentlich: Die Emergenz des Politischen Selbst in Social Media“. In: Paulitz, Tanja/Carstensen, Tanja (Hrsg.): Subjektivierung 2.0. Machtverhältnisse digitaler Öffentlichkeiten. Wiesbaden: Springer VS, S. 101120.

Whitson, Jennifer R. (2014). „Foucault's Fitbit: Governance and Gamification“. In: Walz, Steffen P./Deterding, Sebastian (Hrsg.): The Gameful World. Approaches, Issues, Applications. Cambridge: MIT Press, S. 339-358.

Wiede, Wiebke (2014): „Subjekt und Subjektivierung“. In: Dokupedia-Zeitgeschichte. Begriffe, Methoden und Debatten der zeithistorischen Forschung. URL: http://docupedia.de/zg/Subjekt_und_Subjektivierung [Abruf am: 21.09.2019]. 
